\title{
Rifabutin-associated hypopyon uveitis and retinal vasculitis with a history of acute myeloid leukemia
}

\author{
Wendy M. Smith • Madhu G. Reddy • \\ Kelly A. Hutcheson • Rachel J. Bishop • H. Nida Sen
}

Received: 19 December 2011 / Accepted: 11 January 2012 /Published online: 4 February 2012

(C) The Author(s) 2012. This article is published with open access at SpringerLink.com

\begin{abstract}
Purpose This study reports a case of bilateral rifabutinassociated uveitis in a child with a history of acute myeloid leukemia.

Methods We utilized a clinical case description and brief discussion.

Results A 17-year-old girl presented with acute bilateral anterior uveitis, a hypopyon in the left eye, and moderate bilateral vitritis. She had a history of acute myeloid leukemia status post-allogeneic hematopoietic stem cell transplant 5 years earlier. She was receiving rifabutin for a biopsy-proven $M y$ cobacterium avium complex pulmonary infection. Work up for infectious and neoplastic etiologies was negative. The uveitis initially responded to topical corticosteroids, but recurred when the drops were tapered. Fluorescein angiography demonstrated diffuse vasculitis of small retinal vessels and cystoid macular edema. After rifabutin was discontinued, the uveitis and vasculitis slowly resolved.

Conclusion Fluorescein angiography demonstrated widespread retinal vasculitis which is a rare manifestation of rifabutin-associated uveitis.
\end{abstract}

W. M. Smith $\cdot$ M. G. Reddy $\cdot$ H. N. Sen $(\bowtie)$

Laboratory of Immunology, National Eye Institute,

National Institutes of Health,

10 Center Dr., Building 10, Rm. 10N112,

Bethesda, MD 20892, USA

e-mail: senh@nei.nih.gov

\section{R. J. Bishop}

Consult Services Section, National Eye Institute,

National Institutes of Health,

10 Center Dr., Building 10, Rm. 10C432A,

Bethesda, MD 20814, USA

\section{K. A. Hutcheson}

Department of Ophthalmology,

Children's National Medical Center,

Washington, DC, USA
Keywords Uveitis · Rifabutin · Retinal vasculitis · Cystoid macular edema

\section{Introduction}

Rifabutin is a semisynthetic derivative of rifampin that is effective for the prophylaxis and treatment of Mycobacterium avium complex (MAC) infection. It is frequently used in immunocompromised patients with human immunodeficiency virus (HIV) or a history of organ or stem cell transplantation. Anterior uveitis and hypopyon are well-recognized and frequently reported complications of rifabutin treatment; however, posterior involvement is relatively rare $[1,2]$. Here, we describe a case of rifabutinassociated panuveitis in a young patient with a history of acute myeloid leukemia.

\section{Case report}

A 17-year-old Eritrean girl presented with a 5-day history of redness, tearing, and photophobia which started in her right eye (OD) and then involved her left (OS). She recalled similar, but less severe symptoms over the 2 months prior to presentation. Her medical history was significant for acute myeloid leukemia diagnosed 5 years earlier. She was treated with chemotherapy and received an allogeneic hematopoietic stem cell transplant 6 months after diagnosis. She developed graft-versus-host-disease (GVHD) of the gastrointestinal tract, lungs, and skin with mild ocular surface involvement, but these issues were stable at the time of presentation to the eye clinic.

On initial examination, pinhole visual acuity was 20/32 in each eye (OU), and intraocular pressures were $14 \mathrm{mmHg}$ 
OU. Small non-granulomatous keratic precipitates were present OU. The anterior chambers had $3+$ and $4+$ cells (OD and OS, respectively), and bilateral moderate vitritis was noted. The left eye also had a hypopyon (less than $1 \mathrm{~mm}$ ) and posterior synechiae of the iris (Fig. 1). Both eyes were treated with topical prednisolone acetate $1 \%$ every hour and cyclopentolate twice daily. Due to the concern for recurrent leukemia or infection, aqueous humor was obtained from the left eye; cytology was negative for malignant cells, and cultures were negative. Laboratory testing was negative or within normal limits for RPR, syphilis IgG, hepatitis $\mathrm{B}$ and $\mathrm{C}$, angiotensin-converting enzyme, lysozyme, ANA, anti-dsDNA, rheumatoid factor, and HLAB27. The uveitis resolved after 2 weeks of intensive topical corticosteroids.

Topical corticosteroids were tapered to every 2 hours OU. Ten days later, the patient developed recurrent bilateral anterior uveitis and vitritis with a hypopyon OS. Fluorescein angiography (FA) showed diffuse retinal vascular leakage and late staining of the optic nerves in both eyes (Fig. 2a, b). A more detailed review of her medications and medical history revealed she was on rifabutin for recurrent MAC. Nearly 2 years before she developed uveitis, she had completed a 9month course of ethambutol, clarithromycin, and rifabutin for MAC cultured from a lung biopsy. During the same period, she was on systemic immunosuppressive medications (mycophenolate mofetil, cyclosporine, and prednisone) for GVHD. Eight months before presenting with hypopyon uveitis, ethambutol, clarithromycin, and rifabutin were restarted for recurrent MAC. Rifabutin dosing was twice as high (300 mg every Monday, Wednesday, and Friday), and she was no longer on systemic immunosuppressive agents. After rifabutinassociated uveitis was diagnosed, her medication was switched from rifabutin to moxifloxacin. Topical corticosteroids were continued at the same frequency (every 2 hours), and the hypopyon resolved 5 days later. In

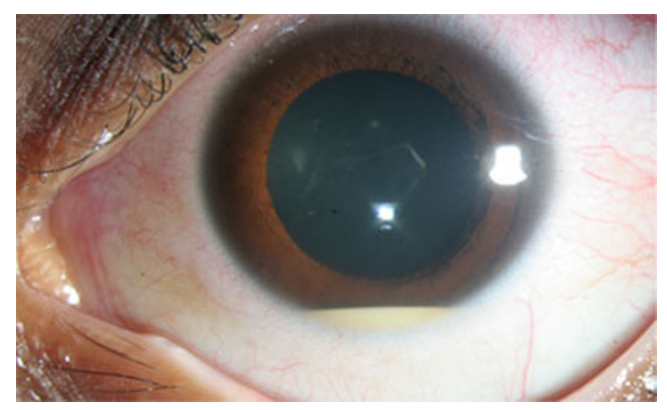

Fig. 1 Anterior segment photograph of the left eye showing a small hypopyon and visible vitreous debris (posterior synechiae were broken by dilation) the first 2 weeks of follow-up, the macular edema initially worsened, OS > OD, but over time, the thickening gradually returned to normal (Fig. 3). On fluorescein angiography, the diffuse retinal leakage had improved at 5 weeks and had resolved after 4 months on a slow topical corticosteroid taper (Fig. 2c-f).

\section{Discussion}

Anterior uveitis and hypopyon are the most frequent manifestations of rifabutin-associated uveitis; however, severe cases may develop dense vitritis with large yellow-white opacities or panuveitis resembling endophthalmitis [3, 4]. Despite the strong association with rifabutin, infectious, autoimmune and neoplastic etiologies should still be investigated depending on the clinical situation. Examination of aqueous humor and/or vitreous via culture, cytology, or polymerase chain reaction can help rule out infection or malignancy.

Although mild to moderate vitritis frequently develops in rifabutin-associated uveitis, posterior involvement is less frequent [5-7]. Similar to the previous cases, our patient presented with uveitis several months after the start of rifabutin and responded quickly to topical corticosteroids and discontinuation of rifabutin. Of the four cases described by Skolik, only one patient had diffuse vasculitis on FA; the others had mild peripheral vasculitis or perivascular sheathing. Of note, the patient with diffuse FA changes was immunocompetent, whereas the other patients had acquired immunodeficiency disease syndrome (AIDS) and CD4 counts less than 100 cells/ $\mathrm{mm}^{3}$. In each of the single case reports by Arevalo and Vaudaux, the rifabutin-associated uveitis was unilateral and the posterior findings were focal. Arevalo's patient had AIDS and a CD4 count of 3 cells $/ \mathrm{mm}^{3}$; a focal area of sclerosed retinal vessels was noted after intraocular injections of vancomycin and gentamicin for presumed endophthalmitis. In the case from Vaudaux, the patient had been treated with systemic prednisone for chronic obstructive pulmonary disease 4 months prior to developing uveitis, but was otherwise immunocompetent. The FA and ocular coherence tomography (OCT) confirmed unilateral cystoid macular edema. In our immunocompetent patient, the vasculitis was notable for its diffuse and bilateral nature and slow resolution after the discontinuation of rifabutin.

Rifabutin-associated uveitis was initially described in HIV-positive patients, but it may also occur in patients who are immunocompetent [7]. Only a few pediatric cases have been reported [8]; however, due to the possible association with low body weight [2], weight-based dosing has been suggested as a strategy to prevent 
Fig. 2 Fluorescein

angiography montages of the right and left eyes: a, b Baseline (prior to discontinuation of rifabutin). c, d Five weeks after stopping rifabutin. e, f Four months after stopping rifabutin
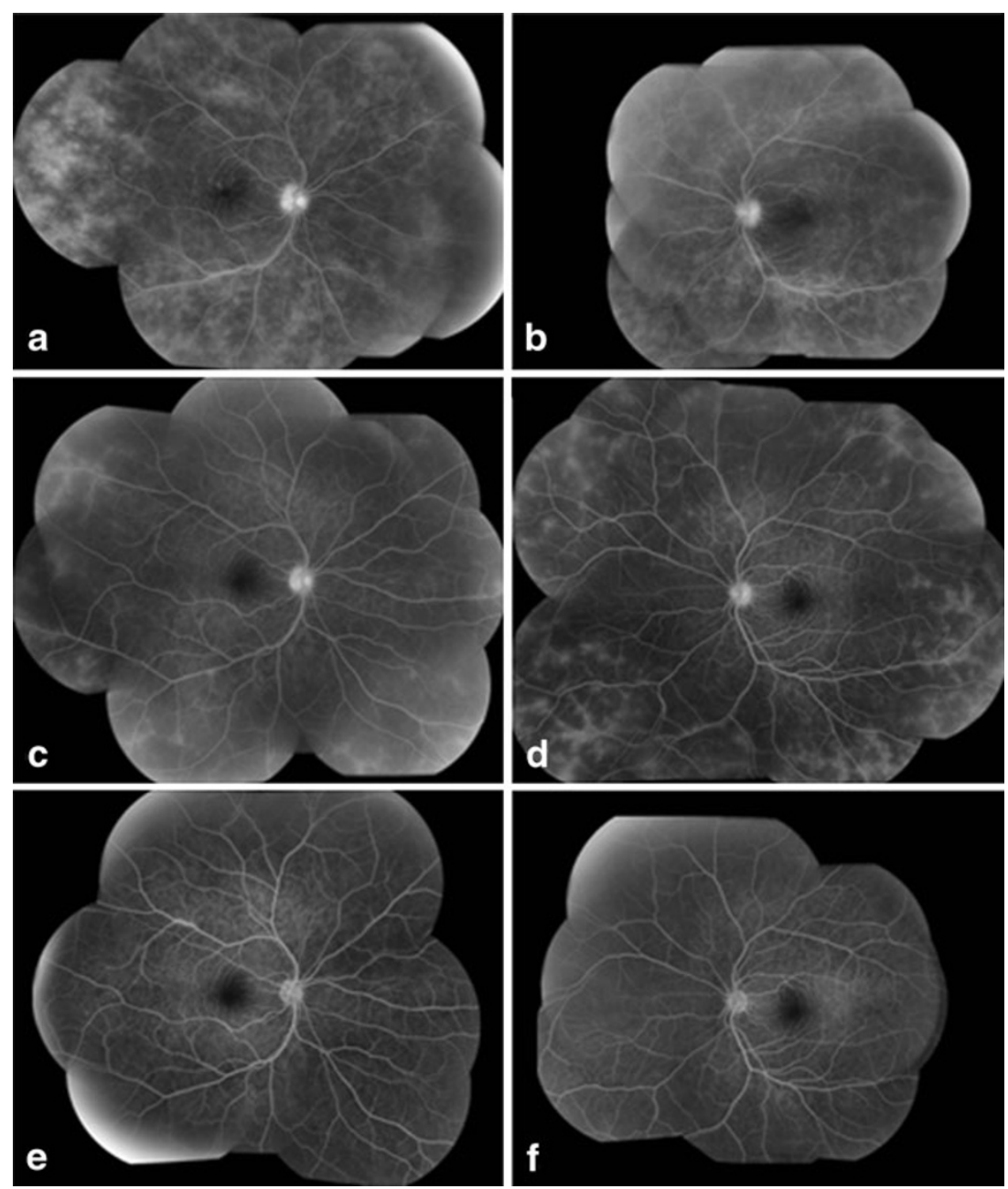

Fig. 3 Cirrus OCT of the left eye: Cystoid macular edema present 9 days (a) after stopping rifabutin that resolved 1 month later (b)
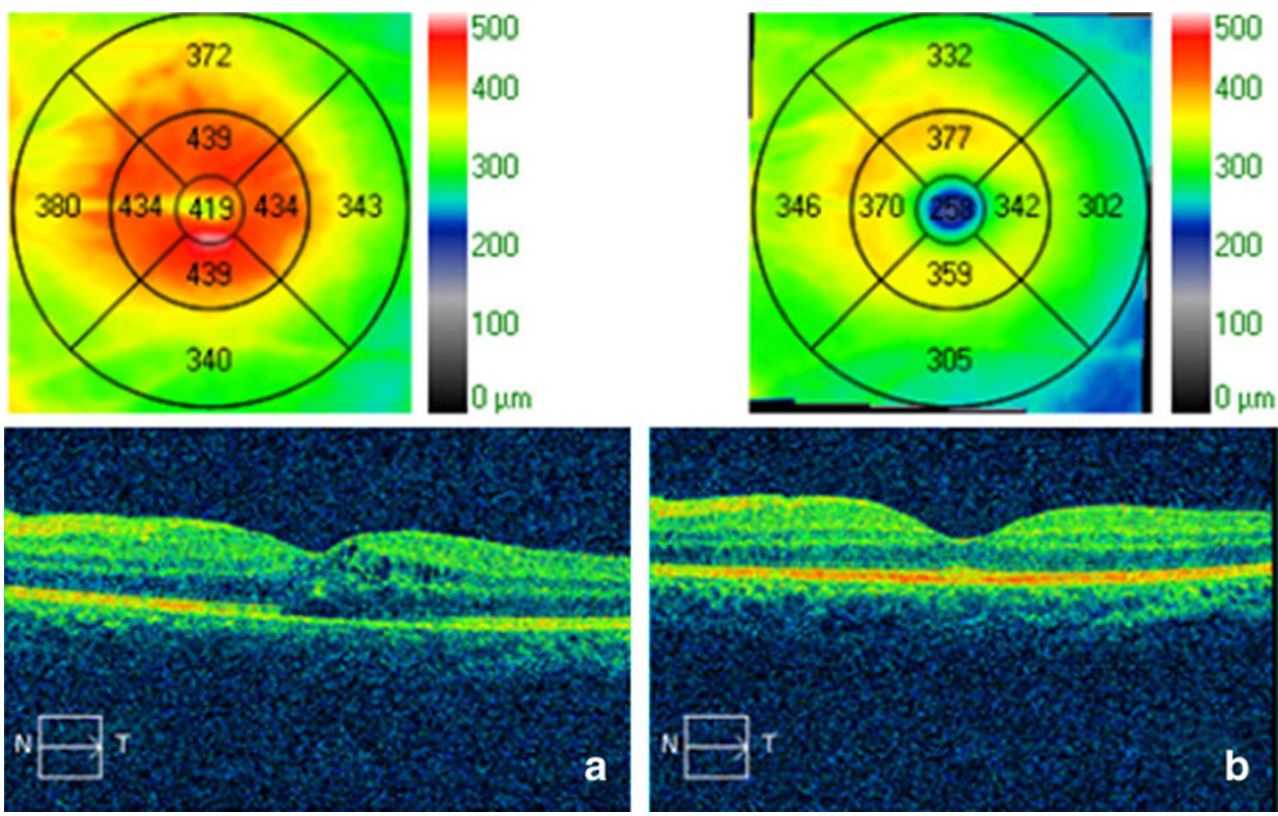
uveitic complications. Our patient weighed $42.5 \mathrm{~kg}$, and uveitis developed after the rifabutin was restarted at twice the initial dose. It is unclear whether the lack of systemic immunomodulatory medications influenced the onset of uveitis during the second course of rifabutin since patients have developed rifabutin uveitis while iatrogenically immunosuppressed after transplantation [9]. Perhaps an intact immune system predisposed our patient to develop more extensive retinal vasculitic changes.

\section{Conclusion}

We describe a case of rifabutin-associated hypopyon uveitis in a young immunocompetent patient with an unusual degree of diffuse bilateral retinal vasculitis. While the anterior chamber and vitreous inflammation disappeared rapidly after discontinuation of rifabutin, resolution of the retinal vasculitis took several months. Fluorescein angiography helped assess the extent and severity of uveitis as well as guide the topical corticosteroid taper.

Acknowledgments This research was supported by the Intramural Research Program of the National Institutes of Health and the National Eye Institute.

Open Access This article is distributed under the terms of the Creative Commons Attribution License which permits any use, distribution, and reproduction in any medium, provided the original author(s) and the source are credited.

\section{References}

1. Jacobs DS, Piliero PJ, Kuperwaser MG, Smith JA, Harris SD, Flanigan TP, Goldberg JH, Ives DV (1994) Acute uveitis associated with rifabutin use in patients with human immunodeficiency virus infection. Am J Ophthalmol 118(6):716-722

2. Shafran SD, Singer J, Zarowny DP, Deschênes J, Phillips P, Turgeon F, Aoki FY, Toma E, Miller M, Duperval R, Lemieux C, Schlech WF 3rd (1998) Determinants of rifabutin-associated uveitis in patients treated with rifabutin, clarithromycin, and ethambutol for Mycobacterium avium complex bacteremia: a multivariate analysis. Canadian HIV Trials Network Protocol 010 Study Group. J Infect Dis 177(1):252-255

3. Rifai A, Peyman GA, Daun M, Wafapoor H (1995) Rifabutinassociated uveitis during prophylaxis for Mycobacterium avium complex infection. Arch Ophthalmol 113(6):707

4. Chaknis MJ, Brooks SE, Mitchell KT, Marcus DM (1996) Inflammatory opacities of the vitreous in rifabutin-associated uveitis. Am J Ophthalmol 122(4):580-582

5. Arevalo JF, Russack V, Freeman WR (1997) New ophthalmic manifestations of presumed rifabutin-related uveitis. Ophthalmic Surg Lasers 28(4):321-324

6. Vaudaux JD, Guex-Crosier Y (2002) Rifabutin-induced cystoid macular oedema. J Antimicrob Chemother 49(2):421-422

7. Skolik S, Willermain F, Caspers LE (2005) Rifabutin-associated panuveitis with retinal vasculitis in pulmonary tuberculosis. Ocul Immunol Inflamm 13(6):483-485

8. Jewelewicz DA, Schiff WM, Brown S, Barile GR (1998) Rifabutinassociated uveitis in an immunosuppressed pediatric patient without acquired immunodeficiency syndrome. Am J Ophthalmol 125 (6):872-873

9. Bhagat N, Read RW, Rao NA, Smith RE, Chong LP (2001) Rifabutin-associated hypopyon uveitis in human immunodeficiency virus-negative immunocompetent individuals. Ophthalmology 108 (4): $750-752$ 